\title{
ReaR
}

ISNN 19894090

Revista electrónica de AnestesiaR

Febrero 2021

FORMACIÓN MÉDICA

\section{Determinantes del Gasto Cardiaco en Anestesia y Cuidados Intensivos.}

Paz Martín D, Tovar Doncel MS.

Complejo Hospitalario de Toledo.

\section{Resumen}

El objetivo fundamental del sistema cardiovascular es transportar fuentes de energía desde el intestino e hígado, y oxígeno desde los pulmones, a todos los órganos para su metabolismo. No menos importante, es la retirada de los productos de desecho del metabolismo y su conducción hacia pulmones, riñones e hígado para su excreción. En el presente trabajo se profundiza en los determinantes del gasto cardiaco.

\section{Introducción}

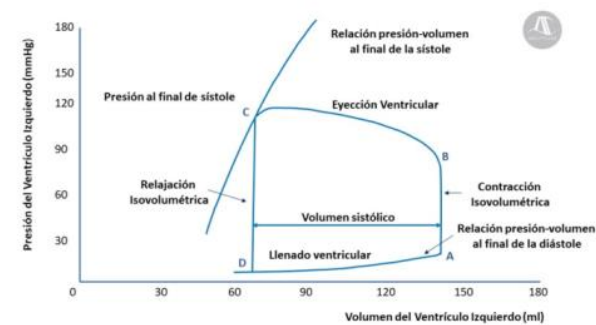

El objetivo fundamental del sistema cardiovascular es transportar fuentes de energía desde el intestino e hígado, y oxígeno desde los pulmones, a todos los órganos para su metabolismo. No menos importante, es la retirada de los productos de desecho del metabolismo y su conducción hacia pulmones, riñones e hígado para su excreción. En el presente trabajo se profundiza en los determinantes del gasto cardiaco.

\section{¿Qué es el Gasto Cardiaco?}

El Gasto Cardiaco (GC) podría definirse como el volumen de sangre eyectado desde el corazón cada minuto, y es un determinante fundamental del transporte de $\mathrm{O} 2$ a los tejidos (DO2).
$\mathrm{GC}(1 / \mathrm{min})=\mathrm{Fc} \times \mathrm{VS}$ donde,

GC gasto cardiaco

Fc frecuencia cardiaca

VS volumen sistólico

En un adulto normal de $70 \mathrm{Kg}$ y en reposo, el GC es de aproximadamente 5-6 L/min (unos 0,07L x $70 \mathrm{lpm}$ ), pudiendo incrementarse a más de 30 $1 /$ min durante el ejercicio extremo. El GC aumenta en proporción al tamaño del individuo, por lo que habitualmente se presenta indexado según área de superficie corporal (Índice Cardiaco), y corresponde al gasto cardíaco por metro cuadrado de superficie corporal. Los valores normales se encuentran entre $2.6-3.2 \mathrm{~L} / \mathrm{min} / \mathrm{m} 2$.

En la tabla 1 se desarrolla la distribución habitual del flujo sanguíneo a los tejidos. 
Tabla 1 Distribución del flujo sanguíneo a los tejidos.

$\begin{array}{llll} & \begin{array}{l}\text { Gasto } \\ \text { Cardiaco } \\ \%\end{array} & \begin{array}{l}\text { Flujo } \\ \mathrm{ml} / \mathrm{min}\end{array} & \begin{array}{l}\mathrm{ml} / \mathrm{min} \\ 100 \mathrm{gr}\end{array} \\ \text { tejido }\end{array}$

\section{¿Qué es gasto cardiaco normal?}

Como se ha expuesto anteriormente, el GC es el producto del volumen sistólico (VS), en ausencia de regurgitación valvular, y la frecuencia cardiaca. El transporte de oxígeno necesita constantemente adaptarse a los requerimientos; aporte vs. demanda. De hecho, la clave fundamental que caracteriza al estado de shock no es la hipotensión (en estadios iniciales del shock, la tensión arterial puede encontrarse normal o elevada debido al aumento de las resistencias vasculares sistémicas), sino la hipoperfusión tisular debida a un disbalance entre el aporte y las necesidades tisulares de oxígeno con la consecuente alteración de la homeostasis orgánica.

Es difícil definir qué es un GC normal en un individuo determinado y en un momento determinado $\mathrm{y}$, desde un punto de vista práctico, tal vez sea más relevante identificar cuando un GC es bajo y se empieza a producir una reducción crítica de la oxigenación tisular. Como tampoco responder a esta cuestión es fácil, existen ventanas clínicas para valorar la perfusión: perfusión cutánea, alteración del estado mental y disminución de la perfusión renal (oliguria). También disponemos de ventanas a la microcirculación como los niveles de lactato sérico (1).
Los principales tres determinantes del VS son: la precarga, la postcarga y la contractilidad. Precarga y contractilidad directamente relacionados con el VS, y la postcarga con una relación inversamente proporcional. Profundicemos en estos conceptos.

\section{Precarga}

La precarga es el principal determinante de la presión ventricular al final de la diástole o presión de llenado.

La precarga se ha descrito en la literatura y en los libros de texto como tensión de las fibras miocárdicas al final de la diástole, longitud de las fibras miocárdicas al final de la diástole, volumen ventricular al final de la diástole, presión de llenado al final de la diástole (2). Sin embargo, actualmente hay consenso en que no es sinónimo de ninguno de estos. En la Figura 1 aparece la relación entre el volumen ventricular izquierdo y la presión intraventricular durante la sístole y la diástole.

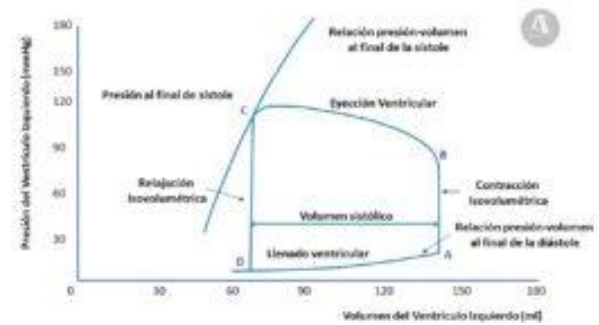

Fig 1. Relación presión-volumen del ventrículo izquierdo durante el ciclo cardiaco. A Cierre de la válvula mitral; B Apertura de la válvula Aórtica (aprox. $80 \mathrm{mmHg}$ ); $\mathrm{C}$ Cierre de la válvula Aórtica; D Apertura de la válvula mitral.

La precarga debería considerarse como una valoración semicuantitativa que engloba todos los factores que contribuyen al estrés (o tensión) pasivo de la pared ventricular al final de la diástole.

Matemáticamente podría expresarse de forma sencilla según la Ley de LaPlace: 
$\mathrm{T}=\mathrm{PR} / 2$ donde,

T: tensión de la pared

P: presión del VI

$\mathrm{R}$ : radio ventricular

Pero dado que el ventrículo es una esfera con paredes gruesas se aplicaría la siguiente fórmula que deriva de la anterior:

$\mathrm{G}=\mathrm{PR} / 2 \mathrm{w}$ donde,

G: estrés de la pared

P: presión

$\mathrm{R}$ : radio

w: grosor de la pared

desarrollando esta última ecuación en el VI:

Gvi=PFDVI . RFDVI/2wvi donde,

PFDVI: Presión al final de la diástole del VI

RFDVI: Radio al final de la diástole del VI

wvi: grosor del VI

De esta ecuación rápidamente se desprende que tanto la presión como el volumen (expresado como radio) del VI, contribuyen a la precarga, pero no son en sí mismos precarga cardiaca.

Curva de Frank-Starling.

La relación entre las presiones de llenado y el VS fue descrita hace un siglo por dos grandes fisiólogos; Otto Frank y Ernest Starling. El corazón presenta una capacidad intrínseca de adaptarse a la cantidad del flujo sanguíneo que vuelve desde las venas hacia la aurícula derecha por minuto (retorno venoso). Cuanto más se distiende el músculo cardíaco durante el llenado, mayor es la fuerza de contracción y mayor es la cantidad de sangre que bombea hacia la aorta. Históricamente se ha considerado que este mecanismo es debido a que los filamentos de actina y de miosina son desplazados hacia un grado cada vez más óptimo de superposición para la generación de fuerza.

Cuando el miocardio se estira por una precarga, cada sarcómero en el miocito se alarga. De forma habitual y a las presiones al final de la diástole normales, la longitud del sarcómero es $\sim 1.8-2.0 \mu \mathrm{m}$, por lo que los miocitos se encuentran en la parte ascendente de la curva de contracción-longitud.

En la figura 2 se representa gráficamente la fuerza contráctil frente a la longitud de sarcómero antes de la contracción. La fuerza máxima se alcanzaría a una longitud de sarcómero de 2.2-2.3 $\mu \mathrm{m}$. Teóricamente, estiramientos más allá de este punto reducirían la fuerza contráctil, sin embargo, en la práctica, in vivo, estas longitudes probablemente nunca se alcanzan porque los miocitos se vuelven muy rígidos por encima de $2.3 \mu \mathrm{m}$.

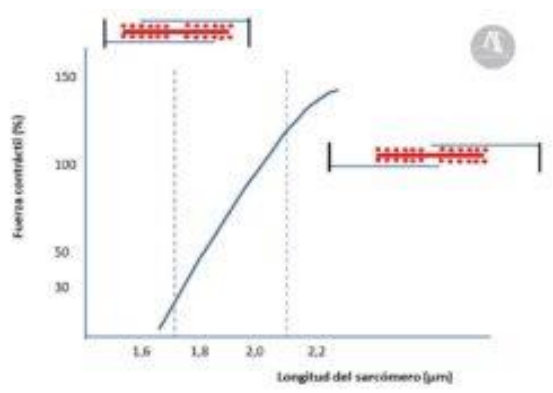

Fig 2. Relación entre la longitud del sarcómero y la contracción miocárdica.

A parte del mecanismo anteriormente descrito, parece que el estiramiento del sarcómero también produciría un incremento de la sensibilidad de los miofilamentos al calcio. 
El fisiólogo estadounidense Arthur Guyton plasmó gráficamente el efecto positivo de la presión venosa central (PVC) en el VS, debido al mecanismo de Frank-Starling (Figura 3). Según esta curva, conforme aumenta la PVC se produce un incremento del GC, y una disminución del flujo del retorno venoso $(\mathrm{RV})$ debido a la reducción de la diferencia de presión entre la presión sistémica media (PSM) y la PVC.

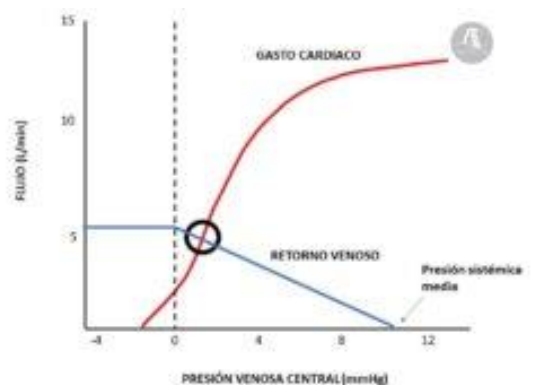

Fig. 3 Control del gasto cardiaco por los vasos sistémicos.

La PSM es el valor de la presión del sistema vascular tras el latido cardiaco (habitualmente en torno a 10-15 $\mathrm{mmHg}$ ), y es la que impulsaría la sangre de nuevo hacia el corazón. La PSM vendría determinada por el volumen vascular estresado (volumen en los vasos sanguíneos que ejerce presión de retorno) y la elastancia vascular.

El RV se define por la siguiente ecuación:

$\mathrm{RV}=\mathrm{PSM}-\mathrm{PVC} / \mathrm{RVeS}$ donde,

\section{$\mathrm{RV}$ retorno venoso}

PSM Presión sistémica media

PVC Presión venosa central

RVeS Resistencia al retorno venoso

Obsérvese en la figura 3, cómo alcanzados unos valores de PVC de 0 , disminuciones posteriores de la PVC no incrementan el $\mathrm{RV}$, probablemente debido a un progresivo colapso de las grandes venas por debajo de la presión atmosférica.

El punto de la gráfica donde se cruzan las curvas representaría la situación ideal en la que el GC y el RV son idénticos.

Por último, conviene recordar que contrariamente a lo que se tiende a pensar (ej. tras las cargas de volumen), el aumento del RV también puede aumentar el GC mediante un incremento de la frecuencia cardiaca. La distensión de la pared de la aurícula derecha produciría un estiramiento del nodo sinusal que aumentaría la frecuencia cardíaca en un 10-20\% (Reflejo de Bainbridge). El estiramiento de la aurícula transmitiría señales a través de los nervios vagos hacia el bulbo raquídeo, y desde aquí de nuevo a través de los nervios vagos y simpáticos se enviarían señales eferentes que aumentarían la frecuencia y la contracción cardíaca.

\section{Postcarga}

La postcarga es la fuerza que se opone a la contracción miocárdica. Se ha estimado, con no demasiado acierto, a través de la presión diastólica de la raíz aórtica, de la presión arterial o de las resistencias vasculares periféricas (Presión arterial media/GC). Realmente correspondería al estrés (o tensión) encontradas por las miofibrillas del VI cuando se contraen contra el volumen al final de la diástole, justo antes de que se produzca el acortamiento. Dado que es complicado medir directamente las fuerzas ventriculares, se emplea una estimación de la postcarga como es la tensión ventricular. De nuevo mediante la Ley de Laplace se describe la relación entre los determinantes de la tensión de la pared ventricular, o postcarga, mediante la ecuación:

$\sigma=(\mathrm{P} \times \mathrm{r}) /(2 \mathrm{w})$, donde 
$\sigma:$ tensión de la pared,

P es la presión de la cámara,

r es el radio de la cámara,

w es el grosor de la pared.

De esta fórmula se deduce que la tensión de la pared es directamente proporcional a la presión ventricular (aumentaría con la elevación de la tensión arterial) y al tamaño (radio), mientras que está inversamente relacionada con el grosor de la pared. Así, la dilatación ventricular aumentaría la postcarga, mientras que la hipertrofia ventricular izquierda (HVI) sería un mecanismo compensatorio que intentaría disminuirla.

\section{Contractilidad}

La contractilidad se define como la capacidad intrínseca de la fibra miocárdica de acortarse independientemente de la precarga y de la postcarga. Por lo tanto, dadas unas condiciones determinadas de éstas últimas, la contractilidad reflejaría la influencia del resto de factores que afectan a la interacción entre las proteínas contráctiles.

Se ve influida por las concentraciones de calcio intracelular, sistema nervioso autónomo, cambios humorales $\mathrm{y}$ agentes farmacológicos.

A pesar de que todo el mundo tiene un concepto intuitivo de qué es contractilidad, la interrelación de todos los factores que la afectan dificulta la propuesta de una definición sencilla.

Se han desarrollado índices de contractilidad cardiaca basados en:

- La presión del VI (PVI): PVI al final de la diástole, la máxima derivada de la PVI (dP/dtmax), pendiente de la relación presiónvolumen ventricular al final de la sístole (Ees) y la $\mathrm{dP} / \mathrm{dt} \max$ dividida por la presión isovolumétrica instantánea.

- El volumen del VI (VVI): VVI al final de la diástole, el VS y la fracción de eyección.

- Análisis de las curvas de presión-volumen: relación presión volumen al final de la sístole, $\mathrm{dP} / \mathrm{dt}$ max vs volumen al final de la diástole (3).

Lo cierto es que no se ha definido un índice clínico completamente satisfactorio e independiente de la precarga y de la postcarga. Opciones razonables serían:

- La medición del punto de presión-volumen tras el cierre de la válvula aórtica (Figura 4 Punto C). Con un aumento de la contractilidad, pero manteniendo la precarga constante se produciría un desplazamiento hacia la izquierda del bucle de relación presión volumen; desde un volumen al final de la diástole determinado (Punto $\mathrm{A}$ ) se llegaría a un volumen al final de la sístole menor (Punto $\mathrm{C}^{\prime}$ ) debido a un mayor volumen sistólico derivado del aumento de la fuerza contráctil.

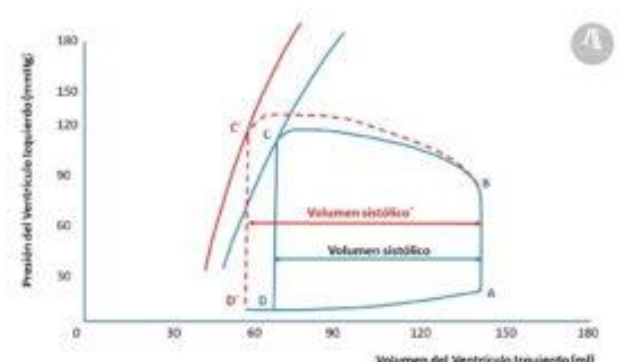

Fig. 4 Efectos del aumento agudo de la contractilidad en la relación presión-volumen ventricular. 
- El análisis del ascenso de la presión ventricular izquierda durante la contracción isovolumétrica (Figura 5) La pendiente de la rama ascendente de la curva indica la velocidad máxima de desarrollo de fuerza por el ventrículo, y su tangente, también denominada $\mathrm{dP} / \mathrm{dt}$ máxima, mostraría la tasa máxima de cambio en la presión a lo largo del tiempo. Los valores normales de $\mathrm{dP} / \mathrm{dt}$ máxima se encuentran alrededor de $1600 \mathrm{mmHg} / \mathrm{s}$, asociándose a contractilidad miocárdica deprimida valores por debajo de $1200 \mathrm{mmHg} / \mathrm{s}$.

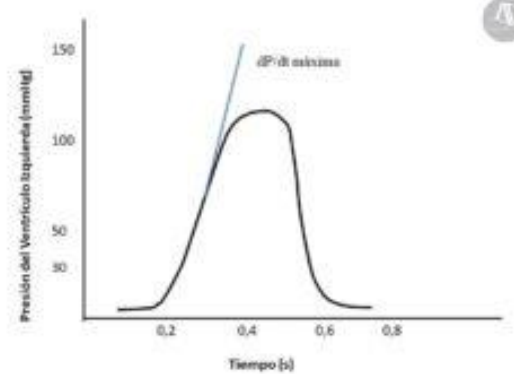

Fig. 5 Presión ventricular izquierda durante la contracción isovolumétrica. $\mathrm{dP} / \mathrm{dt}$ máxima.

\section{Bibliografía}

1. Vincent JL, De Backer D. Circulatory shock. N Engl J Med. 2013;369:172634. (NEJM)

2. Norton JM. Towards consistent definitions for preload and afterload. Adv Physiol Educ. 2001;25:53-61. (HTML)

3. Naiyanetr P. (2013) A Review of Cardiac Contractility Indices during LVAD Support. In: Long M. (eds) World Congress on Medical Physics and Biomedical Engineering May 2631, 2012, Beijing, China. IFMBE Proceedings, vol 39. Springer, Berlin, Heidelberg (HTML)

Correspondencia al autor

Daniel Paz Martín

dpaz@anestesiar.org

MD PhD EDAIC EDIC. Servicio Anestesiología y Reanimación.

Complejo Hospitalario de Toledo. Sección de Cuidados Intensivos de la SEDAR

Aceptado para el blog en julio de 2020 